\title{
Equilibrium Asset Prices and Savings of Heterogeneous Agents in the Presence of Incomplete Markets and Portfolio Constraints
}

\author{
Albert Marcet* \\ and \\ Kenneth J. Singleton ${ }^{\dagger \ddagger \S}$ \\ July 1998 \\ (First Version, April 1990)
}

*Universitat Pompeu Fabra (Barcelona).

†Stanford University and NBER.

¥We have benefited from helpful discussions with Darrell Duffie, Rody Manuelli and David Marshall. Research support from from the National Science Foundation, DGICYT (Spanish Ministry of Education), CIRIT (Catalan Conselleria of Education), CREF (Universitat Pompeu Fabra) and the Financial Research Initiative at Stanford University is greatly appreciated.

$\S$ Forthcoming in Macroeconomic Dynamics. 
Mailing addresses:

\author{
Albert Marcet \\ Departament d'Economia i Empresa \\ Universitat Pompeu Fabra \\ Ramon Trias Fargas, 25-27 \\ 08005 Barcelona \\ Spain \\ albert.marcet@econ.upf.es
}

\author{
Kenneth J. Singleton \\ Graduate School of Business \\ Stanford University \\ Stanford, CA-94305 \\ U.S.A. \\ ken@future.stanford.edu
}

\section{Abstract}

We study the quantitative properties of a dynamic general equilibrium model in which agents face both idiosyncratic and aggregate income risk, state-dependent borrowing constraints that bind in some but not all periods and markets are incomplete. Optimal individual consumption-savings plans and equilibrium asset prices are computed under various assumptions about income uncertainty. Then we investigate whether our general equilibrium model with incomplete markets replicates two empirical observations: the high correlation between individual consumption and individual income, and the equity premium puzzle. We find that, when the driving processes are calibrated according to the data from wage income in different sectors of the US economy, the results move in the direction of explaining these observations, but the model falls short of explaining the observed correlations quantitatively. If the incomes of agents are assumed independent of each other, the observations can be explained quantitatively.

Keywords: incomplete markets, credit constraints, equity premium puzzle, consumption volatility, simulation. JEL classification: E21, G11, G12. 


\section{Preamble}

We started working on this paper in the late 80's; when we were both on the faculty of Carnegie Mellon University. Many papers, at the time, explored the empirical implications of consumption-based equilibrium asset pricing models (many tried to explain the risk-premium puzzle, many tested the model by GMM), and they all maintained the assumption of homogeneous agents and/or complete markets. It seemed to us that the next natural step in this branch of literature would be to explore the implications of equilibrium, incomplete market economies with heterogeneous agents. Such a model is a candidate for solving the equity premium puzzle, since agents are limited in their ability to smooth consumption risks. Perhaps more interestingly, a number of additional issues can be studied, such as the relationship between individual consumption and income, the behavior of asset trading, equilibrium portfolio decisions, the distribution of wealth, etc. The new methods for simulating non-linear rational expectations equilibrium models made the exploration of these issues feasible.

We adapted the parameterized expectations approach $P E A$ to solve heterogeneous agents models with portfolio constraints, and then studied the properties of our model using the sample moments of simulated consumptions, incomes, and asset prices and holdings of the agents. Since then, other papers have studied similar models by simulation; some of the authors are A. Bernardo, J. Coleman, W. den Haan, J. Diaz, J. Heaton, M. Hugget, K. Judd, P. Krusell, D. Lucas, E. McGrattan, F. Obiols, A. Smith and C. Telmer. Some of these papers have used, explored, or criticized our algorithm; also, Christiano and Fisher discussed our way of dealing with occasionally binding inequality constraints. The current paper is a shortened version of our June 1991 working paper which was distributed, for example, at the NBER summer institute. It includes a new concluding Section 5 that discusses some unresolved issues. We have not included an extensive review of the literature written since our 1991 draft. Rather, in the spirit of the "vintage paper" series in Macroeconomic Dynamics, we are simply reporting a condensed version of our ideas as they had developed up to the time we postponed work on this project. Since then, the literature on models with heterogeneous agents and incomplete markets has grown substantially and more is now known about the properties of these models.

In preparing the last version of the paper, back in 1991, we encountered the following problem: when we refined the solution to increase the accuracy, or when we tried to solve the model with different initial conditions, the algorithm required many iterations to find a fixed point, often wandered through a wide region of the admissible space of choice variables, and sometimes settled down at different laws of motion. Some of the properties of the model 
were practically insensitive to changing initial conditions. For example, this was true of the asset prices and the correlations of individual consumptions and incomes. However, some other properties of the model, such as the levels of asset holdings by each agent, were notably different. We explored different reasons why this might be happening, some of which are discussed in more detail in section 5. To this date, we feel we do not fully understand the reasons for these apparent sensitivities, and will be more comfortable with the results when more is understood about the theoretical properties of economies with incomplete markets. Specifically, we would like to know more about the circumstances under which there is a unique equilibrium and when this equilibrium is an ergodic process.

Some of the findings in this paper have been confirmed for alternative specifications of preferences and frictions in financial markets. For instance, other researchers have confirmed that active trading in a small number of securities often allows agents to approximately achieve the consumption plans of the complete-markets equilibrium. And even with state-dependent portfolio constraints, it is difficult to generate a large equity premium. Our basic specification of trading opportunities and sources of uncertainty (two assets held in equilibrium, three driving processes with continuous distributions, non-symmetric agents) remains among the richer models examined, so we suspect that more than minor changes to preferences or the constraint sets will be necessary to resolve the empirical puzzles that motivated this study.

\section{Introduction}

In this paper we study the quantitative properties of a dynamic general equilibrium model in which agents face both idiosyncratic and aggregate income risk, markets are incomplete, and in the presence of state-dependent borrowing constraints that bind in some, but not all, periods. Optimal individual consumption-savings plans and equilibrium asset prices are computed under various assumptions about income uncertainty. Then we investigate whether our general equilibrium model with incomplete markets replicates some relations between consumptions, incomes, and asset returns observed in micro and aggregate data better than a representative agent model.

Specifically, we are motivated by two observations: the correlation of individual consumption and individual income, and the equity premium puzzle. For the first of these observations, evidence from various microeconomic data bases suggests that the consumptions of individual agents are more highly correlated over time with their own incomes than with aggregate income 
(see, e.g., Carroll and Summers (1991)). ${ }^{1}$ Furthermore, the consumptions of agents who hold relatively large quantities of common stocks appear to be more highly correlated with stock returns than consumptions of those agents who do not hold stocks (Mankiw and Zeldes (1990)). These observations are contrary to what might be expected from optimal consumption smoothing by risk averse agents in an economy with complete markets. We explore whether these observations can be explained in general equilibrium by the combined effects of market incompleteness and portfolio constraints on savings and consumption decisions.

In addition, we investigate the properties of asset returns and the equity premium in our incomplete markets economies. Mehra and Prescott (1985) show that simple representative agent models imply excess returns on equity that are substantially smaller than those observed historically. Furthermore, the empirical evidence from the analysis of Euler equations with much richer specifications of preferences suggests that a large class of representative agent models are not consistent with the observed behavior of asset returns and consumption. ${ }^{2}$ Weil (1989) argues that the equity premium puzzle can be interpreted equally as a risk-free-rate puzzle in that the mean riskless rate implied by a large class of representative agent models is too large relative to the mean computed from historical data. In the context of a static model with idiosyncratic income risk, Mankiw (1986) illustrates that incomplete markets can potentially explain the large equity premium and return volatility. In this paper we re-examine these issues in the context of a dynamic general equilibrium model under different parameterizations.

To move away from the representative agent model and/or complete markets framework, we assume there are two types of consumers with idiosyncratic and exogenous incomes from labor. We permit trading in both a riskless real bond and a risky equity. This provides some risk sharing opportunities, but not enough to complete the markets in equilibrium so financial markets are incomplete. In addition, we assume there are limits to the amount of assets that agents can hold; in particular, we impose borrowing constraints on bond holdings and short-sale constraints on equity holdings.

An important precursor to our analysis of consumption-savings relations is the study by Deaton (1989). He studied a partial equilibrium model of

\footnotetext{
${ }^{1}$ The empirical evidence from macroeconomic data (Flavin (1981)) and microeconomic data (Hall and Mishkin (1982) and Zeldes (1989)) suggests that changes in consumption are positively related to predictable changes in income. This evidence, though not obtained directly from models which accommodate restrictions on borrowing, has also been interpreted as a manifestation of borrowing constraints.

${ }^{2}$ See Singleton (1993) for a review of the empirical evidence on representative agent, intertemporal asset pricing models.
} 
impatient agents trading a single asset with a constant, exogenous interest rate, and facing a non-negativity constraint on wealth. Our framework differs from his in several potentially important respects. In addition to having more than one agent and more than one asset, consumptions and asset prices in our model are jointly determined in general equilibrium. If the asset holdings of a substantial fraction of the population approach the constraints, then equilibrium interest rates will adjust, thereby affecting the extent to which constraints actually bind. This endogenous adjustment of asset prices is absent from models, such as Deaton's, which assume asset prices are fixed or exogenously given. Of course, such models also preclude an analysis of the effects of portfolio constraints on the properties of asset returns. ${ }^{3}$

The computational aspects of our analysis are complementary to the studies by Cox and Huang (1987), Karatzas, Lehoczky, and Shreve (1989), He and Pearson (1989a,b), and Breeden (1989), among others. The papers by He and Pearson and Breeden, in particular, derive optimal portfolio rules for an individual agent facing incomplete markets and short-sale constraints. All of these studies are partial equilibrium in nature in that asset prices are taken as state variables and then the optimum problem of an individual agent is solved. In contrast, we present an algorithm for computing the equilibrium asset prices simultaneously with the consumption and portfolio policies of heterogeneous agents facing incomplete markets and subject to portfolio constraints.

The details of the model are described in Section 2. An algorithm for solving economic models in which agents are subject to portfolio constraints is described in section 3. We solve the model using the parameterized expectations approach (PEA). ${ }^{4}$ In Section 3, we discuss in detail some issues involved in applying PEA to this model and, in particular, some complications arising from the presence of debt limits and the need to solve for the optimal holdings of two assets. The algorithm can be easily adapted to different specifications of the utility function, types of agents, and financial market structure.

In Section 4, we investigate the distributions of consumptions, incomes, and asset holdings implied by the model presented in section 2. The income and dividend processes are calibrated by fitting a trivariate vector autoregression using data from the incomes of workers in the durable and nondurable

\footnotetext{
${ }^{3}$ A concurrent study by Telmer (1990) examines the implications of incomplete markets for the riskfree rate puzzle in the context of a model in which agents trade a riskless bond, but do not face portfolio constraints. In contrast to Telmer and Deaton, our model incorporates the economically plausible constraint that the levels of borrowing permitted are functions of the agents' income levels.

${ }^{4}$ See Marcet (1989), den Haan and Marcet (1990) and Marcet and Marshall (1994).
} 
manufacturing sectors and aggregate per-capita dividends for the U.S. during the period 1947:1 through 1988:4. The standard deviations of the income shocks are then scaled to reflect the higher variability of individual incomes compared to estimates from aggregated data reported in the microeconomics literature (e.g., MaCurdy (1982)). In this manner, we obtain a benchmark parameterization for the exogenous processes. The model is solved for the benchmark case and for several variations of that case, as well as for various changes in the form of the portfolio constraints. We find that our benchmark model moves in the right direction in terms of explaining the two puzzles from the data that we discuss above. Nevertheless, from a quantitative standpoint, the two puzzles are not solved: through intensive buying and selling of financial claims (as contrasted to reliance on the payoffs of asset portfolios that would happen under complete markets) agents achieve consumptions similar to what would be achieved with complete financial markets, and the resulting risk premium is small. We show, however, that for certain income processes, our incomplete markets economy with state-dependent borrowing constraints reproduces the observations cited above: individual consumptions are more highly correlated with own rather than aggregate incomes, and the average levels of both the riskless interest rate and the equity premium (4.6\%) are consistent with the historical evidence.

\section{Model Specification and the Existence of an Equilibrium}

In this section we present an illustrative economy with two classes of agents and a market structure that precludes complete insurance arrangements against idiosyncratic income shocks. The size of the population is constant and normalized to one, with type 1 agents constituting a fraction $\pi$ and type 2 agents constitute a fraction (1- $\pi$ ). All agents are assumed to be price takers in goods and securities markets. We also assume that there are three sources of a single, nonstorable consumption good: the exogenous dividend flows of a Lucas (1978)-style tree and the exogenous endowments of the two types of agents. The number of shares representing claims to the dividend stream is normalized to one. Thus, letting $y_{i t}$ denote the endowment of a type $i$ agent and $d_{t}$ denote the per-share dividend, the aggregate endowment is

$$
y_{t}^{*}=\pi y_{1 t}+(1-\pi) y_{2 t}+d_{t}
$$

and the market clearing condition for the consumption good is

$$
\pi c_{1 t}+(1-\pi) c_{2 t}=y_{t}^{*}
$$


where $c_{i t}$ denotes consumption at date $t$ by agent $i$. The endowment vector $y_{t}=\left(\ln y_{1 t}, \ln y_{2 t}, d_{t}\right)^{\prime}$ is assumed to follow the Markov process

$$
y_{t}=h\left(y_{t-1}, \varepsilon_{t}, \eta_{0}\right)
$$

where $\left\{\varepsilon_{t}\right\}$ is an i.i.d. process with $E\left[\varepsilon_{t}\right]=0$ and $E\left[\varepsilon_{t}^{\prime}\right]=I$, and $\eta_{0}$ is a vector of parameters. The process $\left\{y_{t}\right\}$ is assumed to have a stationary distribution. In particular, we assume that there is no growth in the endowments or population.

There are two securities traded in this economy: an equity-like security and a one-period bond. An equity share is a claim to the future dividend stream $d_{t}$ with price $p_{t}^{s}$. Share holdings of the $i^{\text {th }}$ agent are denoted by $a_{i t}$. (Recall that the outstanding number of shares is normalized to 1.) The bond pays one unit of consumption in the subsequent period with certainty, is in zero net supply, and it is traded at an equilibrium price $p_{t}^{b}$ in a competitive market. The bond holdings of agent $i$ are denoted by $b_{i t}, i=1,2$. The market clearing conditions for the assets at each period $t$ are:

$$
\begin{aligned}
& \pi a_{1 t}+(1-\pi) a_{2 t}=1 \\
& \pi b_{1 t}+(1-\pi) b_{2 t}=0
\end{aligned}
$$

A type $i$ agent is assumed to choose consumption and investment plans so as to maximize the objective function:

$$
E\left(\sum_{t=0}^{\infty} \delta_{i}^{t} u_{i}\left(c_{i t}, \gamma_{i}\right) \mid I_{0}\right)
$$

where $\gamma_{i}$ is a parameter vector governing the period utility function, $I_{0}$ is the common information set of agents at date 0 , and $0<\delta_{i}<1$ is the subjective discount factor of type $i$ agents. Utility is maximized subject to the budget constraints

$$
c_{i t}+p_{t}^{s} a_{i t}+p_{t}^{b} b_{i t}=a_{i t-1}\left(p_{t}^{s}+d_{t}\right)+b_{i t-1}+y_{i t}
$$

In order for the maximization problem of the agents to be well defined we have to introduce additional constraints on the agents' investment plans. Otherwise, agents could obtain arbitrarily high levels of consumption in any period by borrowing (more precisely, by selecting very negative bond holdings) and repaying these loans with additional borrowing in subsequent periods. Consequently, accumulated debt holdings could become so large that their future stream of endowments would not be sufficiently large to repay this debt. To preclude such Ponzi schemes, the following constraints are imposed on the asset holdings of the agents:

$$
b_{i t} \geq K_{i}^{b}\left(x_{t}\right)
$$




$$
a_{i t} \geq K_{i}^{s}\left(x_{t}\right)
$$

for all $t, i$, where $K_{i}^{b}$ is typically negative, and $x_{t}$ is the vector of states in the model, to be specified below. These constraints can take many forms including fixed negative numbers and limitations on borrowing based on an agent's recent income history.

We assume full information: both agents at time $t$ know the whole history of past shocks, so that their equilibrium choices $\left(c_{i t}, a_{i t}, b_{i t}\right)$ are contingent on realizations of $I_{t} \equiv\left\{y_{t-s}: s>0\right\}$. We consider equilibria where this information set can be summarized by a low-dimensional state vector. Clearly, the state vector must include at least the information in $\left(y_{t},\left(a_{i t-1}, b_{i t-1}\right)_{i=1,2}\right)$, since these are the variables that appear in the constraints of agents. From (3) and (4) it is clear that, once $\left(b_{1 t-1}, a_{1 t-1}\right)$ are known, $\left(a_{2 t-1}, b_{2 t}\right)$ carry no additional information and they can be dropped from the state vector. Furthermore, the only relevant information about asset holdings that matters for the decision of agent $i$ at time $t$ is his total wealth, $w_{i t}=a_{i t-1}\left(p_{t}^{s}+d_{t}\right)+b_{i t-1}$. Thus, we choose as the state vector for this model $x_{t}=\left(w_{1 t}, y_{t}\right)$, and we make the choice of both agents at time $t$ contingent only on $x_{t}$. A larger number of classes of agents are also easily accommodated by including the wealth levels of more agents in $x_{t}$.

In our economic environment, trading in the claims to the exogenous dividend stream and the one-period bonds is not sufficient for agents to fully hedge their consumption risk. There are three sources of uncertainty (two idiosyncratic incomes and dividend shocks) and each is drawn from a continuous distribution so, in principle, the number of Arrow securities that are needed to complete the markets is equal to the cardinality of $R^{3}{ }^{5}$ The admittedly arbitrary restriction of security selection in our model provides an interesting economic environment for exploring the consequences of portfolio constraints for savings and asset prices, while maintaining sufficient tractability to permit various properties of the solution algorithm to be explored. ${ }^{6}$

To our knowledge, sufficient conditions for the existence of a stationary Markov equilibrium for this economy have not yet been established. However,

\footnotetext{
${ }^{5}$ Bossaerts and Green (1990) show that if agents have constant relative risk averse preferences with common discount factors and risk aversion parameters, then two assets are sufficient to complete the market: a perpetuity (infinitely lived bond) and the market portfolio. However, this two-fund allocation will in general not emerge from our model, because there are three sources of income uncertainty in our model and agents cannot trade claims to their own income streams- effectively, they are prevented from holding the relevant "market" portfolio. In addition, the bonds in our model are single period, not infinitely lived.

${ }^{6}$ Using the techniques developed in this paper, Ketterer and Marcet (1990) explore the implications for risk sharing arrangements of adding an option on the aggregate dividend for a special case of the models examined subsequently.
} 
the results in Duffie, Geanakoplos, Mas-Colell, and McClennan (1994) show that an equilibrium does exist for discrete approximations to our economy. Specifically, they assume that the preferences of the $i^{\text {th }}$ agent are bounded above and unbounded below, the portfolio holdings are subject to lower bounds, and the exogenous state space is discrete. The preference assumption is satisfied, for example, by the members of the HARA class examined in Section 4 (assuming the aggregate endowment has bounded support) and in many previous studies of consumption and asset returns. In addition, for the special case of state-independent debt limit $K \equiv\left(K_{1}^{s}, K_{2}^{s}, K_{1}^{b}, K_{2}^{b}\right)$, equations (7) and (8) provide lower bounds on the levels of debt and short sales. Though $\varepsilon_{t}$ in our simulated economy has a continuous distribution, the Markov model (2) can be approximated arbitrarily well by a discrete state, Markov process (e.g., Tauchen (1985)). The Duffie, et. al. existence result applies directly to this approximate model. ${ }^{7}$

The properties of the equilibria of models with state-dependent portfolio constraints are less well understood than the case of fixed constraints. If $K$ is a function of the exogenous state vector $y$ alone and the distribution of $y$ has compact support, then the previous remarks about existence of an equilibrium continue to apply. On the other hand, if $K$ depends on lagged asset holdings, it is not immediate that the budget set of the agents is compact; in principle, asset holdings could grow without bound in this case, even though at each date the levels of $a$ and $b$ are constrained to lie in a compact set with a boundary predetermined by the values of lagged asset holdings and $y_{t}$. The class of economies with state-dependent portfolio constraints for which an equilibrium exists has not been characterized in the literature. We proceed (without formal proof) under the assumption that an equilibrium to this economy exits and that the equilibrium prices and consumptions are time-invariant functions of the state vector.

The first-order conditions of a type $i$ agent's problem are given by the constraints they face plus the following Kuhn-Tucker conditions for equity holdings:

either

$$
\begin{aligned}
& \operatorname{mu}_{i}\left(c_{i t}, \gamma_{i}\right) p_{t}^{s}=\delta_{i} E_{t}\left[\mathrm{mu}_{i}\left(c_{i t+1}, \gamma_{i}\right)\left(p_{t+1}^{s}+d_{t+1}\right)\right] \\
& \text { or } \\
& \operatorname{mu}_{i}\left(c_{i t}, \gamma_{i}\right) p_{t}^{s} \geq \delta_{i} E_{t}\left[\mathrm{mu}_{i}\left(c_{i t+1}, \gamma_{i}\right)\left(p_{t+1}^{s}+d_{t+1}\right)\right] \text { and } a_{i t}=K_{i}^{s}\left(x_{t}\right)
\end{aligned}
$$

\footnotetext{
${ }^{7}$ The solution algorithm described in section 3 for models with binding constraints on portfolio holdings can be applied without modification to models based on discrete state-space approximations to continuous-state Markov forcing processes.
} 
and, for bond holdings:

either

$$
\begin{aligned}
& \operatorname{mu}_{i}\left(c_{i t}, \gamma_{i}\right) p_{t}^{b}=\delta_{i} E_{t}\left[\operatorname{mu}_{i}\left(c_{i t+1}, \gamma_{i}\right)\right] \text { and } b_{i t}>K_{i}^{b}\left(x_{t}\right), \\
& \text { or } \\
& \operatorname{mu}_{i}\left(c_{i t}, \gamma_{i}\right) p_{t}^{b} \geq \delta_{i} E_{t}\left[\operatorname{mu}_{i}\left(c_{i t+1}, \gamma_{i}\right)\right] \text { and } b_{i t}=K_{i}^{b}\left(x_{t}\right)
\end{aligned}
$$

where $\mathrm{mu}_{i}\left(c_{i t}, \gamma_{i}\right)$ denotes the marginal utility of consumption at date $t$. In periods when (9) and (10) are satisfied for a type $i$ agent, the credit and short-sale constraints are non-binding and we have the usual Euler equations for bonds and equity. When $\left(9^{\prime}\right)$ or $\left(10^{\prime}\right)$ is satisfied, at least one of the constraints is binding and the associated Euler equation is satisfied only as an inequality.

Our goal is to solve for the eight unknown variables $\left.\left\{\left(c_{i t}, a_{i t}, b_{i t}\right)_{i=1,2}, p_{t}^{s}, p_{t}^{b}\right)\right\}$ given initial conditions. These variables have to satisfy the two budget constraints (6), the three market clearing conditions (1) - (4), and the Euler equations (9), (10) (or the alternative equations $\left(9^{\prime}\right),\left(10^{\prime}\right)$ ). It would appear that there are eight unknowns and nine equations per period, but a version of Walras' Law applies and one of the budget constraints can be dropped from the calculations at each period.

In practice, there are two obstacles to solving these equations. First, the Euler equations involve conditional expectations which must be computed as functions of $x_{t}$ in a way that the rational expectations hypothesis is satisfied. This problem has been solved in the context of representative-agent business cycle models by using approximations to the expectations, several of which are described in Taylor and Uhlig (1990) and the references therein. Second, we face the additional complication that the constraints (7) or (8) will in general be binding in some periods, so that $\left(9^{\prime}\right)$ or $\left(10^{\prime}\right)$ will hold in those periods. In the next section we extend the parameterized expectations approach to solving stochastic growth models to accommodate models with heterogeneous agents subject to portfolio constraints.

\section{An Algorithm for Computing the Equilib- rium of the Model}

\subsection{PEA}

We first present a general description of the $P E A^{8}$. This algorithm works by iteratively approximating the conditional expectations that often appear in

\footnotetext{
${ }^{8}$ This description and notation follows Marcet and Marshall (1994).
} 
equilibrium conditions under rational expectations. In our model, expectations appear in (9) - (10). Specifically, consider a model that satisfies

$$
g\left(E_{t}\left[\phi\left(z_{t+1}\right)\right], z_{t}, z_{t-1}, y_{t}\right)=0
$$

for all $t$, where $g: R^{m} \times R^{n} \times R^{n} \times R^{s} \rightarrow R^{q}$ and $\phi: R^{n} \rightarrow R^{m}$ are known functions. The $n$-dimensional vector $z_{t}$ includes all endogenous variables in the model. The exogenous process $y_{t}$ is assumed to be Markov of order one. Also, given a set of state variables $x_{t}=f\left(z_{t}\right)$ for a known function $f: R^{n} \rightarrow R^{l}$ we compute a solution such that

$$
E_{t}\left[\phi\left(z_{t+1}\right)\right]=E\left[\phi\left(z_{t+1}\right) \mid x_{t}\right]=\mathcal{E}\left(x_{t}\right)
$$

for a time-invariant function $\mathcal{E}$.

To find a solution $\left\{z_{t}\right\}$ that satisfies (11) and (12) we need to find an approximation to $\mathcal{E}$. We proceed by choosing a class of functions that can approximate any function arbitrarily well, and we fix the degree of approximation. For example, we can choose polynomials of a third degree. Then, for a third degree polynomial with given coefficients we simulate the system and find the third order polynomial with the highest predictive power. We look for a polynomial of third degree that generates simulations such that this polynomial is, precisely, the one with the highest predictive power. This is a candidate to be a good approximation to the solution because the conditional expectation function $\mathcal{E}$ has an analogous property in that, the solution generated from substituting $\mathcal{E}$ in (11) is such that this is the function that has the highest predictive power. Obviously, to obtain arbitrary accuracy, we would need to drive the degree of approximation to infinity ${ }^{9}$

Formally, the algorithm is expressed as follows:

Step 1 : Write the system (11) in such a way that $g$ is invertible with respect to its second argument. Select a set of state variables $x$. Consider a function $\Psi(\beta, \cdot): R^{l} \rightarrow R^{m}$, with $\beta \in R^{\nu \times m}$ such that, for $\nu$ large, we can approximate any function $F: R^{l} \rightarrow R^{m}$ arbitrarily well. The $i$-th element of $\Psi$ is given by $\psi_{i}\left(\beta_{i}, \cdot\right)$, is meant to approximate $E_{t}\left(\phi_{i}\left(z_{t+1}\right)\right)$, where $\beta_{i}$ is the $i$-th column of $\beta$.

Replace the true conditional expectations by the finitely-parameterized function $\Psi$ to obtain

$$
g\left(\Psi\left(\beta, x_{t}(\beta)\right), z_{t}(\beta), z_{t-1}(\beta), y_{t}\right)=0
$$

Notice here that $\left\{z_{t}(\beta)\right\}$ is the value of the endogenous variables if, instead of using the conditional expectations, agents used $\Psi(\beta, \cdot)$ to

\footnotetext{
${ }^{9}$ See Marcet and Marshall (1994) for a formal proof of convergence.
} 
make their forecasts. Fix $y_{0}$ and $z_{0}$. Draw a sample of size $T$ of the exogenous stochastic shock $\left(y_{1}, \ldots, y_{T}\right)$ for large $T$.

Step 2 : For a given $\beta \in R^{\nu \times m}$, recursively calculate $\left\{z_{t}(\beta)\right\}_{t=1}^{T}$ using (13) and the realization of $y$ drawn in step 1 . It follows that $z_{t}(\beta)$ has the Markov representation $z_{t}(\beta)=H\left(z_{t-1}(\beta), y_{t} ; \beta\right)$. We have to insure that $H(\cdot ; \beta)$ generates an ergodic process in order for the next Step to make sense, and in order to use the simulated moments as approximations to the true moments of the model ${ }^{10}$.

Step 3 : Consider the mapping $G_{T}: R^{\nu \times m} \rightarrow R^{\nu \times m}$ whose columns are defined by

$$
G_{T, j}(\beta)=\arg \min _{\xi \in R^{\nu}} \frac{1}{T} \sum_{t=0}^{T}\left(\phi_{j}\left(z_{t+1}(\beta)\right)-\psi_{j}\left(\xi, x_{t}(\beta)\right)\right)^{2}
$$

that is, if agents use $\beta$ to make their predictions, then $\psi\left(G_{T, j}(\beta), \cdot\right)$ yields the best forecast of $\phi_{j}\left(z_{t+1}\right)$ (within the function considered).

Step 4 : Find the fixed point

$$
\beta_{f}=G_{T}\left(\beta_{f}\right)
$$

The approximate solution is given by $\left\{z_{t}\left(\beta_{f}\right)\right\}_{t=1}^{T}$, our approximation to $\mathcal{E}$ is given by $\Psi\left(\beta_{f}, \cdot\right)$, and our approximate law of motion is given by $H\left(\cdot ; \beta_{f}\right)$.

\subsection{Applying PEA to our model (Steps 1 and 2).}

Our model is the special case of (11) with $z_{t} \equiv\left(\left(c_{i t}, a_{i t}, b_{i t}\right)_{i=1,2}, p_{t}^{s}, p_{t}^{b}\right)$ and with the system of eight equations $g$ in (11) given by four Euler equations (with Kuhn-Tucker conditions), one budget constraint, and three market clearing conditions. It is clear that $\phi$ is given by

$$
\begin{aligned}
& \phi_{i}\left(z_{t+1}\right) \equiv \operatorname{mu}_{i}\left(c_{i t+1}, \gamma_{i}\right)\left(p_{t+1}^{s}+d_{t+1}\right) \\
& \phi_{2+i}\left(z_{t+1}\right) \equiv \operatorname{mu}_{i}\left(c_{i t+1}, \gamma_{i}\right) .
\end{aligned}
$$

for $i=1,2$. A key element of Step 1 is to insure that $g$ is invertible with respect to its second argument, otherwise, Step 2 can not be performed. We will see later that we need to modify $g$ in order to have invertibility.

\footnotetext{
${ }^{10}$ One way to check for ergodicity is to impose the AUC condition of Duffie and Singleton (1993) on $H(\cdot ; \beta)$.
} 
To apply Step 1 , we take $x_{t} \equiv\left(w_{1 t}, y_{t}\right)$. Also, we choose as $\Psi$ a set of four exponentiated polynomials, so that the $i$-th parameterized expectation for the case $\nu=1$ is $^{11}$

$$
\psi_{i}\left(\beta_{i}, x_{t}\right) \equiv \exp \left(\beta_{i 1}+\left(\beta_{i 2}, \beta_{i 3}, \beta_{i 4}\right) \log y_{t}+\beta_{i 5} w_{1 t}\right) .
$$

This functional form guarantees that the value of this function is positive, consistent with $\phi$ being strictly positive. Furthermore, it is easy to see that this parameterization is exactly correct in the case of homogeneous agents with log-normally distributed $y$ and only one bond. We will use the notation $P i_{t}^{s}$ and $P i_{t}^{b}$ to refer to the parametric approximations to the expectations appearing in the stock and bond equations respectively for a type $i$ agent, e.g., $P 1_{t}^{b} \equiv \psi_{3}\left(\beta_{3}, x_{t}\right)$ and so on.

Next, consider Step 2, which consists of solving for $z_{t}(\beta)$ in (13). Because of the Kuhn-Tucker conditions, we have to consider several cases, so let us start with

\section{Case 1: Unconstrained Asset Holdings}

If the choices of both agents are unconstrained, then (9) and (10) are both satisfied. Dividing each of these equations for $i=1$ by the same equation for $i=2$, we get

$$
\frac{\mathrm{mu}_{1}\left(c_{1 t}, \gamma_{1}\right)}{\mathrm{mu}_{2}\left(c_{2 t}, \gamma_{2}\right)}=\frac{\delta_{1} P 1_{t}^{s}}{\delta_{2} P 2_{t}^{s}}
$$

and

$$
\frac{\mathrm{mu}_{1}\left(c_{1 t}, \gamma_{1}\right)}{\mathrm{mu}_{2}\left(c_{2 t}, \gamma_{2}\right)}=\frac{\delta_{1} P 1_{t}^{b}}{\delta_{2} P 2_{t}^{b}}
$$

These equations together with (1) provide three equations to solve for the two unknowns $\left(c_{1 t}, c_{2 t}\right)$. Obviously, the system is over-determined, and we can not find solutions for consumptions from these equations. ${ }^{12}$

The problem here is that, the way we have written $g$ initially, it is not invertible with respect to the endogenous variables, as required by Step 1. We need to rewrite an equivalent system that satisfies this invertibility requirement. Toward this end, consider any positive and increasing function $\mathcal{G}: R \rightarrow R_{++}$. It is easy to check that equations (9)-(9') for $i=1$ are equivalent to:

either

\footnotetext{
${ }^{11}$ For higher degrees of approximation (higher $\nu$ 's) a higher degree polynomial in the variables $\left(\log y_{t}, w_{1 t}\right)$ would appear inside 'exp'.

${ }^{12}$ Imposing $P 2_{t}^{s} \equiv\left(P 2_{t}^{b} P 1_{t}^{s}\right) / P 1_{t}^{b}$ in the parameterized expectations would allow us to solve for consumption, but then we would loose one equation, we would not be able to solve for the asset prices, and the system $g$ would then be underdetermined.
} 


$$
\begin{aligned}
p_{t}^{s} \mathcal{G}\left(a_{1 t}^{s}\right)=\delta E_{t}\left(\frac{\mathrm{mu}_{1}\left(c_{1 t+1}, \gamma_{1}\right)}{\mathrm{mu}_{1}\left(c_{1 t}, \gamma_{1}\right)}\left(p_{t+1}^{s}+d_{t+1}\right) \mathcal{G}\left(a_{1 t}^{s}\right)\right) & \\
& \text { and } a_{1 t}>K_{1}^{s}\left(x_{t}\right)
\end{aligned}
$$

or

$$
\begin{aligned}
p_{t}^{s} \mathcal{G}\left(a_{1 t}^{s}\right) \geq \delta E_{t}\left(\frac{\mathrm{mu}_{1}\left(c_{1 t+1}, \gamma_{1}\right)}{\operatorname{mu}_{1}\left(c_{1 t}, \gamma_{1}\right)}\left(p_{t+1}^{s}+d_{t+1}\right) \mathcal{G}\left(a_{1 t}^{s}\right)\right) & \\
& \text { and } a_{1 t}=K_{1}^{s}\left(x_{t}\right) .
\end{aligned}
$$

So, we replace the equity conditions (9)-(9') for $i=1$ by (19)-(19') when defining $g$ and $\phi$. For completeness, the revised system of equations is presented in Appendix 1.

Now, we can perform Step 2 when the debt limits are not binding as follows. Combining (18) and (1) we obtain

$$
\frac{\mathrm{mu}_{1}\left(c_{1 t}, \gamma_{1}\right)}{\mathrm{mu}_{2}\left(\frac{y_{t}^{*}-\pi c_{1 t}}{1-\pi}, \gamma_{2}\right)}=\frac{\delta_{1} P 1_{t}^{b}}{\delta_{2} P 2_{t}^{b}}
$$

which is a single nonlinear equation that can be used to solve for the unknown $c_{1 t}$. Each remaining variable can be calculated recursively in one additional step: $c_{2 t}$ is given by (1), $p_{t}^{b}$ and $p_{t}^{s}$ are given by the parameterized versions of (10), and (9) for a type 2 agent, $a_{1 t}$ is found from (19), and $b_{1 t}$ is calculated from the budget constraint (6). The asset holdings for $i=2$ are determined directly from the market clearing conditions for assets.

\section{Case 2: Constrained Asset Holdings}

The preceding computations presume that no agent is constrained in choosing asset holdings. Let $\bar{z}_{t}$ denote the solution obtained in Case 1 . If either $\bar{a}_{i t}$ or $\bar{b}_{i t}$ are outside their bound, Case 1 can not be the solution for that period. Suppose, for example, that we obtain $\bar{a}_{1 t}<K_{1}^{s}\left(x_{t}\right)$. It means that the inequality in (19) for $i=1$ is not satisfied and we must impose (19') instead. Then, the equality $a_{1 t}=K_{1}^{s}\left(x_{t}\right)$ determines asset holdings in that period. Notice that we have 'lost' one equation, since the Euler equation is not satisfied as an equality, but we have gained the equation $a_{1 t}=K_{1}^{s}\left(x_{t}\right)$, and we still have eight equations to solve for eight unknowns. The remaining variables are found using similar steps as in the unconstrained system.

In the case that $\bar{b}_{1 t}<K_{1}^{b}\left(x_{t}\right)$ we have to use (10') instead of (10) for $i=1$. To this end, first set $\bar{b}_{1 t}=K_{1}^{b}\left(x_{t}\right)$, then obtain stock prices and holdings from the stock Euler equations and, combining (10) for agent 2 with the budget constraint (6) and market clearing condition (1) we obtain

$$
c_{1 t}+a_{1 t} p_{t}^{s}+b_{1 t} \frac{\delta_{2} P 2_{t}^{b}}{\mathrm{mu}_{2}\left(\frac{y_{t}^{*}-\pi c_{1 t}}{1-\pi}, \gamma_{2}\right)}=a_{1 t-1}\left(p_{t}^{s}+d_{t}\right)+b_{1 t-1}+y_{1 t},
$$


which determines $c_{1 t}$. The remaining variables are once again easily computed.

In following this procedure, one must assure that the inequality conditions of the Kuhn-Tucker conditions are satisfied. That this is indeed the case can be seen as follows. Consider the situation that, when solving under Case 1, we find that $\bar{b}_{1 t}<K_{1}^{b}\left(x_{t}\right)$. Then we have to check that, when we shift to Case 2, the inequality in (10'):

$$
\mathrm{mu}_{1}\left(c_{1 t}, \gamma_{1}\right) p_{t}^{b} \geq \delta_{1} P 1_{t}
$$

holds. But if Case 1 yields $\bar{b}_{1 t}$ that are too low, bonds of agent 1 have to go up in Case 2, hence $\bar{c}_{1 t}>c_{1 t}$, so that $\mathrm{mu}_{1}\left(c_{1 t}, \gamma_{1}\right)>\mathrm{mu}_{1}\left(\bar{c}_{1 t}, \gamma_{1}\right)$ and $\mathrm{mu}_{2}\left(c_{2 t}, \gamma_{2}\right)<\mathrm{mu}_{2}\left(\bar{c}_{2 t}, \gamma_{2}\right)$. Since (10) is satisfied for $i=2$, we have that $p_{t}^{b}>$ $\bar{p}_{t}^{b}$. This implies that $\mathrm{mu}_{1}\left(c_{1 t}, \gamma_{1}\right) p_{t}^{b}>\mathrm{mu}_{1}\left(\bar{c}_{1 t}, \gamma_{1}\right) \bar{p}_{t}^{b}=\delta_{1} P 1_{t}$ unambiguously and (22) is satisfied. A similar argument works for the equity Kuhn-Tucker conditions, although in that case it is essential that we have an increasing $\mathcal{G}$.

Summarizing, to calculate the endogenous variables for a given parameterized expectation $z_{t}(\beta)$ in each period, we first solve the model as if all agents are unconstrained by the limits on portfolio transactions. Then, if an agent borrows too much or takes too large of a short position in equity, the relevant asset holdings are set at their limits. We have to go down through all possible cases (each agent being constrained in each possible asset), until one is found where all the conditions are satisfied. It turns out that, by proceeding in this manner, all the inequalities implied by the Kuhn-Tucker conditions are satisfied automatically.

To perform Step 3, notice that, in our model, we have four Euler equations, each with one expectation, so that $m=4$. This means that, in Step 3 , we have to solve four problems of the form of (14) to obtain $G_{j T}$ for $j=1, \ldots, 4$. For this purpose we just compute a non-linear regression of $\phi_{j}\left(z_{t+1}(\beta)\right)$ on $\psi_{j}\left(\cdot, x_{t}(\beta)\right)$ for each $j$. Details on how to run this regression can be found in den Haan and Marcet (1990).

In order to find a fixed point $\beta_{f}=G_{T}\left(\beta_{f}\right)$ a number of iterative schemes could be used. In practice, we use the following updating scheme

$$
\beta^{\tau}=\mu G_{T}\left(\beta^{\tau-1}\right)+(1-\mu) \beta^{\tau-1}
$$

for some $\mu \in(0,1)$ and some initial condition $\beta^{0}$. The choice of $\mu$ near zero forces the algorithm to take small steps.

Notice that, once Steps 3 and 4 are completed, the possibility of being constrained in the future affects agents' decisions today, even if the debt 
limits are not binding in the current period. This is because $\beta_{f}$ is selected so as to predict certain functions of future variables, future variables might be affected by the debt limits, so future debt limits influence $\beta_{f}$ and, therefore, today's decisions.

One potential difficulty with $P E A$ is that if $\beta$ takes a value where the difference equation implied by (13) is unstable at some point along the iterations, the simulations in Step 2 will be explosive. In order to avoid this problem, we use homotopy ideas to move gradually from a known solution to the desired solution. Since the $\beta_{f}$ for bonds and the representative agent model is easy to find, we move from the homogeneous agent equilibrium to heterogeneous agent case by making small changes in the parameters of the model. That is, we introduce heterogeneity in small steps until we reach the desired model.

We choose $\mathcal{G}(a)=\exp \{a\}$ in (19) and we set $\mu=.2$ in (23). In solving for $c_{1 t}$ in (20) for iteration $\tau$ we use values for consumption found at iteration $\tau-1$ as initial conditions, so that this non-linear equation (which is solved $T$ times per iteration) is solved in one or two steps. Finally, the simulation size $T$ in the definition of $G_{T}$ was set at 9600 .

Whenever we are interested in a certain moment $E\left(m\left(z_{t}\right)\right)$ at the stationary distribution for certain parameter values of the utility functions and forcing processes, after obtaining $\beta_{f}$ consistent with these parameters, we approximate this moment by

$$
M_{T}=\frac{1}{T} \sum_{t=1}^{T} m\left(z_{t}\left(\beta_{f}\right)\right) .
$$

Since we only consider solutions that have a stationary distribution, computing this moment with a large enough $T$ gives a good approximation to the desired moment. For this purpose, we check that $H\left(\cdot ; \beta_{f}\right)$ satisfies the AUC condition of Duffie and Singleton (1993), so as to guarantee that the simulated moments converge to the desired moment for any initial condition.

\section{Optimal Consumption and Portfolio Deci- sions for Illustrative Economies}

In this section we present some quantitative evidence on the effects of portfolio constraints on consumptions and asset prices in the context of the model set forth in Section 2. Since agents will take into account the possibility that these constraints may bind in future periods, the constraints themselves need not bind with high frequency in order to have a significant impact on current 
economic behavior. The magnitudes of these effects are investigated in the context of models calibrated to macro and micro economic data.

The instantaneous utility function $u_{i}(c)$ is assumed to be $u_{i}(c)=c^{\gamma_{i}+1} /\left(\gamma_{i}+\right.$ 1). The exogenous income and dividend processes are assumed to be distributed as log-normal $A R(1)$

$$
\log y_{t}=B+A \log y_{t-1}+\varepsilon_{t}
$$

where $\varepsilon_{t}$ is a three-dimensional i.i.d. process with distribution $N(0, \Sigma)$.

In order to simulate our model we need to assume values for the parameters $A, B, \Sigma$, preferences, and the borrowing and short-sale constraints. Given the large number of free parameters, a thorough investigation of the properties of the equilibria of our model over the entire admissible parameter space is infeasible. Instead, we choose one benchmark set of parameters calibrated so as to make some moments close to those implied by certain macro and micro economic data sets. We fix $\gamma_{i}=-2$. and $\delta_{i}=.99$ for $i=1,2$ in all our models, so this discount factor corresponds to an average real riskless interest rate of about $4 \%$ per annum.

In order to calibrate the process for the exogenous variables we interpret agents of type 1 as workers in the durable goods sector and agents of type 2 as workers in the non-durable goods sector of the U.S. economy. These sectors were chosen because of the differing cyclical variability of wages; hence, our simulations illustrate how agents with different income processes trade assets in order to smooth consumption. Dividends from the equity-like securities were taken to be aggregate per-capital dividend income. Historical data was obtained from the Citibase data file for the sample period 1947 to 1988 .

Using this data, we fit a trivariate, first-order autoregression with the variables ordered with durable and non-durable sector incomes followed by dividend income. The estimates indicate a very high autocorrelation in these aggregate series and very little feedback between variables. On the other hand the innovations of the income series are highly correlated. After setting to zero all of the elements of $A$ that are not significantly different from zero at the $5 \%$ level and selecting the point estimates of the parameters that are significantly different from zero we choose the following value of $A$ :

$$
A=\left[\begin{array}{ccc}
.85 & .15 & .0 \\
.0 & .95 & .0 \\
.0 & .0 & .95
\end{array}\right]
$$

As Deaton (1989) observed, the high persistence in the income processes may weaken agents' desire for consumption smoothing in equilibrium, because smoothing consumption over long cycles requires more assets and more sacrifice of consumption than with low or negative autocorrelation coefficients. We shall return to this issue after displaying our results. 
The covariance matrix of the innovations is also taken from the regression results, except that this matrix is scaled up by a factor of three. The reason for scaling was to make the variances of the individual incomes closer to those reported in the microeconomic literature. MaCurdy (1982) estimated the conditional variance of the logarithm of income in an $A R I M A(0,1,1)$ process to be .235 for annual data. Arguing that this estimate is implausibly large due to measurement error in recorded income, Deaton used values between .10 and .15. The quarterly estimates we use for our benchmark model place type 1 agents somewhat below MaCurdy's estimate and type 2 agents at the low end of Deaton's values. Specifically, with standard deviations on the diagonal and correlations on the off-diagonal, our second moment matrix is:

$$
\Sigma=\left[\begin{array}{ccc}
.05 & .5 & .2 \\
.5 & .03 & .2 \\
.2 & .2 & .01
\end{array}\right]
$$

Notice that the incomes of the two types of agents are correlated with each other and with the exogenous dividend process, so that there is both idiosyncratic and aggregate uncertainty from the point of view of each agent.

Finally, the intercept vector $B$ is chosen so that the implied means for the levels of per capita incomes equaled the sample means for our data set: $(41,34)$ for $\left(y_{1}, y_{2}\right)$, and the mean of aggregate per capita dividends was chosen to be 7 , which is approximately $10 \%$ of aggregate income on average. The resulting income and dividend series are scaled so that $y^{*}$ has a mean of unity.

The proportion of type 1 agents in the population is set at $\pi=.6$, which is roughly the proportion of workers in the durable-goods sector in the economy.

The portfolio constraints for the benchmark case are of two forms. First the constraint on stock holdings was fixed at $K_{t}^{s}\left(x_{t}\right)=.0$ shares, which is a no short-selling constraint. As will become apparent from the results, this is a very weak constraint because the average value of the equilibrium price of equity is large relative to average income and, therefore, small changes in share holdings can have substantial effects on consumption. Subsequently, we explore the implications of setting the constraint on equity holdings at .5 shares.

We assume a state-dependent borrowing constraint with $K_{i}^{b}\left(x_{t}\right)=-.33 y_{i t}$. This choice is intended to approximate the commonly imposed income constraint for borrowing from financial institutions that the costs of servicing personal debt do not exceed one-third of personal income. For long-term debt, this constraint usually applies to the repayment of interest and principal. Since the only borrowing in our model is for a single period and the bonds are purchased at discount, this constraint analogously applies to the 
repayment of principal and interest on loans. We explore the effects of relaxing this constraint on savings behavior after examining this benchmark case.

Thus, with these choices of the parameters we construct a benchmark model, which we call Model 1. Then we vary the parameters for the income process and debt limits in several directions to explore a total of seven models. The parameter choices for all the models examined are displayed in Table 1. This Table also contains the correlation of individual incomes implied by the different $A, B, \Sigma$ considered in each model. For each model, 1700 observations were simulated and sample estimates of several moments of consumptions and incomes were computed using the last 1600 of these observations. These moments are displayed in Table 2.

In the complete markets counterpart to our economy, equilibrium consumption allocations satisfy $c_{1 t} / c_{2 t}=\lambda$ for some constant $\lambda$. Hence, one implication of complete markets in this economic environment is that individual consumptions are perfectly correlated with aggregate income. Concurrently, we would expect that the correlation of an agent's consumption with its own income would be much lower (possibly zero). Thus, the extent to which consumptions are not perfectly correlated with aggregate income provides one measure of the departure of the incomplete from the complete markets solutions for this economy.

For our benchmark Model 1, the correlations between aggregate income $y^{*}$ and individual consumptions are less than unity and, hence, the optimal allocations differ from those of the complete markets equilibrium. However, correlations are close to unity, especially for type 1 agents. Furthermore, for both types of agents, the correlations between $c_{i t}$ and $y^{*}$ are larger than the correlations between $c_{i t}$ and $y_{i t}$. In this respect, the departures from a complete markets equilibrium are not large enough to explain the relatively large correlations between individual consumptions and incomes documented in Carroll and Summers (1991).

Figures 1 through 6 display observations some observation from $t=100$ through $t=1000$ for several of the endogenous variables in the benchmark Model 1. As Figure 1 demonstrates, consumption of type 1 agents has both a higher mean and greater variability than consumption of type 2 agents. This is consistent with $y_{1}$ having a larger mean and a larger variance than $y_{2}$.

The bond holdings of type $1(\mathrm{AB})$ and type 2 (AB2) agents are displayed in Figures 2 and 3, along with the state dependent borrowing constraints (con1 and con2, respectively). Bond holdings evidence substantial fluctuation since the bond market is serving as the primary means of risk sharing in this economy. To see more clearly the roles of equity and bonds in risk sharing, the 
bond and stock holdings of type 2 agents are reproduced in Figures 4 and 5 for a subperiod of two hundred observations. The bond holdings displayed in Figure 4 vary substantially both over time and between positive and negative levels. Comparison of the values of AB2 at the beginning, middle, and end of this subperiod shows that type 2 agents tend to be borrowers when their income level is relatively low and lenders when they have relatively high incomes. By market clearing and the fact that the correlation between the two incomes is .78 (Table 1), this implies that type 1 agents have the opposite borrowing-income relation.

The stock holdings of type 2 agents displayed in Figure 5 reveal that type 2 agents hold a smaller share of the equity than type 1 agents. Furthermore, in contrast to the bond holdings, there is relatively little variation in the stock holdings, with the stock holdings AS2 of type 2 agents rising slightly during periods of relatively high individual income levels (displayed as Y2 in Figure 5). Evidently, type 1 agents, with the higher income and consumption levels, tend to smooth their consumption by holding a larger share of the equity (which is less volatile than either income process). Stock prices (PS in Figure 6) tend to be high when incomes and consumptions are high. To sustain their equity holdings during periods of relatively high incomes and equity prices, type 1 agents borrow. Type 2 agents, on the other hand, hold a smaller share of the equity, and they borrow during periods of low income to smooth consumption.

Models 2 and 3 impose the weaker borrowing constraint that borrowing not exceed one-half of the current period income. In the case of Model 2, all other aspects of the specification remain the same as in Model 1. From Table 2 it is seen that relaxing the borrowing constraint does lead to larger correlations between individual consumptions and aggregate income, but the increase is from a base that is already near unity and the increase is small. In Model 3 we relax the borrowing constraint relative to the benchmark model and simultaneously tighten the short-sale constraint by setting $K_{i}^{s}\left(x_{t}\right)=.5$. This leads to a slight decline in the mean equity holdings of type 1 agents and small decline in average income, but again these changes are not notable.

In sum, the results from Models 1 through 3 suggest that the incompleteness of markets due to the restrictions on trading of a bond and an equity imply less than perfect correlations between individual consumptions and aggregate incomes. However, these correlations are greater than .99 for type 1 agents and .95 for type 2 agents. Furthermore, the tightening of the borrowing and short-sale constraints considered has virtually no effect on equilibrium decisions. These findings suggest that markets are effectively nearly complete for the assumed income processes and portfolio constraints.

Though suggested by the autoregressions with macroeconomic data, the 
correlation of .78 between the incomes of representative agents from the two sectors of the U.S. economy is very large. Therefore, in Model 4 we explore the implications of reducing this correlation by setting $A_{12}=0$, which is within the $2 \%$ confidence interval about the point estimate of $A_{12}$. In addition, the correlation of the innovations in the incomes is set at .2 , so that the cross-correlations of the innovations on incomes and dividend series are all the same. These changes lead to a decline in the correlation of $y_{1 t}$ and $y_{2 t}$ to .13 . Perhaps the most striking feature of the results for Model 4 is that the agents' consumptions are now more highly correlated with their own incomes than with aggregate income. For instance, the correlation of a type 2 agent's consumption with aggregate income declines to about .8, while their correlation with own income is about .86. The low correlation between $c_{i t}$ and $y_{t}^{*}$ is also striking and indicative of combined importance of market incompleteness and the borrowing constraints for this economy.

Model 5 is identical to Model 4, except that we fix the borrowing constraints at .33 . To interpret this fixed constraint, recall that the incomes have been normalized so that the mean of $y_{1}$ is unity and the mean of $y_{2}$ is .83. Hence, the fixed constraint for type 1 agents is equal to the mean of the constraint they face under the income-dependent constraint. Similarly, the fixed constraint for type 2 agents is approximately $20 \%$ weaker than the mean constraint faced in the benchmark model. The effective weakening of the borrowing constraints is illustrated graphically in Figure 7 which displays the actual borrowing of type 2 agents for Model 5 and the series CON2 equal to -. $33 y_{2 t}$, which is the constraint under the income-dependent borrowing constraint. As expected, the weakening of the average borrowing constraint for type 2 agents lead to somewhat higher correlations $\rho_{c_{i} y^{*}}$ in Model 5. But overall the results are very similar to those for Model 4 . We conclude that, for the income processes in Models 4 and 5, the income-dependent nature of the constraint does not appear to be a major determinant of our findings. This may well change if the variances of the income processes are increased.

In Model 6 we return to the income-dependent borrowing constraint -.33y and increase the standard deviation of the income process for type 2 agents to the same value as type 1 agents (.05). The higher value of $\sigma_{\varepsilon_{2}}$ implies that $\rho_{y_{1} y_{2}}$ increases to .17, the mean consumptions of type 1 agents fall and type 2 agents increase. In addition, $\sigma_{c_{2}}$ is now substantially larger than $\sigma_{c_{1}}$. The increase in $\sigma_{c_{2}}$ is attributable to the higher volatility in income in the presence of portfolio constraints. With the higher income variance, type 2 agents are more frequently at or near their borrowing constraint (see Figure 8) and, thus, trading in bonds is a less effective means of smoothing consumption.

To compensate for the reduced risk sharing role of bonds, agents increase substantially the average volume of trading in equity (Figure 9). Recall from 
Figure 4 that, in the benchmark model, type 2 agents held about .3 shares, whereas the average holdings in Figure 9 are about .75 shares. Equally interesting is the substantial increase of trading in equity in economy 6 compared to economy 1 . The volume of "liquidity," or non-information based trades is clearly affected by the overall structure of asset markets and portfolio constraints. Furthermore, the effect of market incompleteness on trading volume in equity markets has a business cycle component with volume being higher during periods when the relatively low incomes of type 2 agents preclude the desired level of borrowing to smooth consumption.

Though we match many of the empirical characteristics of the consumption and income series with Model 6, the degree of consumption smoothing accomplished in equilibrium is perhaps implausibly low. For instance, the standard deviation of $y_{1}$ in Model 6 is .099, whereas the standard deviation of $c_{1}$ is .088 . Similarly, the standard deviations of $y_{2}$ and $c_{2}$ are both approximately .13 . Thus, equilibrium consumptions are nearly as volatile as the underlying income processes. As noted during our discussion of the autoregressive matrix $A$, this feature of the model is attributable to the high degree of persistence in the stationary income processes. In subsequent research we plan on exploring the implications for our analysis of real growth in the exogenous income and dividend processes.

Associated with the effects of market incompleteness on savings and consumption decisions are interesting effects on asset returns. Several sample moments involving bond and equity returns are displayed in Table 3. The mean returns are reported on an annualized basis, while the standard deviations and correlations are for the quarterly simulated data. The mean real returns on equity are plausible for our sample period. The standard deviations of equity returns are largest in Models 3 and 6 . In the former case this is due to the tighter short-sale constraint, while in the latter case it is consistent with our earlier observations about trading volume in Model 6 . Ibbotson (1989) reports a standard deviation for real stock returns for the period 1926 through 1988 of $21.1 \%$, so our quarterly number seems in line with the historical data.

Perhaps the most striking moment of asset returns in Table 3 is the mean return on riskless bonds. The mean bond return is over $2 \%$ for all of the models except Model 6 for which it falls to $.8 \%$ (.776\% more precisely). Weil (1989) has argued that the equity premium "puzzle" documented by Mehra and Prescott (1985), among others, is as much a risk free rate puzzle. That is, representative agent models yield mean risk free rates that are much too high relative to historical experience. Mehra and Prescott (1985) report an annual average real riskless return of $.75 \%$ for the U.S., while the corresponding number in Ibbotson (1989) is .5\% . For Models 1 through 5, our estimate of 
the mean bond return is much too high compared to these estimates and, in this respect, shares an important limitation of representative agent, complete markets models.

On the other hand, in Model 6 the estimated mean bond return is comparable to the estimates for U.S. data. What differentiates Model 6 from the other models is that the incomplete risk sharing provided by the equity and bond markets for type 2 agents is relatively acute; type 2 agents are at or near their borrowing constraint with relatively high frequency. Type 2 agents borrow when income is low and lend when income is high and, thus, their stock of bonds serves as a buffer for income fluctuations. Because of the strong precautionary demand for savings, equilibrium bond returns must be sufficiently low on average for both type 1 and type 2 agents to be content with a level of debt for type 2 agents below what they would desire in the absence of borrowing constraints. The source of this high precautionary demand is the relatively high income uncertainties; the average riskless rate is higher in Model 4 where one of the agents has less income uncertainty. We conclude that the combined features of incomplete markets and portfolio constraints are capable of resolving the risk free rate puzzle but, in order to achieve the bond return observed in the data, we need to assume that the individual income processes are less correlated than in our benchmark case given by Model 1.

The sixth column of Table 2 displays the average equity premiums in our models. As anticipated by our discussion of risk free rates, compared with the equity premia usually obtained from versions of complete market models, the premiums generated by these incomplete market economies are quite large. In particular, the premium in Model 6 is $4.6 \%$ per annum. Though below the estimate of $6 \%$ often cited in the literature on the equity premium puzzle, $4.6 \%$ is not out of line with U.S. experience during our sample period. ${ }^{13}$

The last two columns of Table 3 display the correlations between the individual consumptions of agents and the return on equity. Mankiw and Zeldes (1990) have observed that the consumptions of stockholders seem to be much more highly correlated with stock returns than the consumptions of non-stockholders. In our models, both types of agents hold stocks, but type 2 agents hold a much smaller proportion of the outstanding shares. When

\footnotetext{
${ }^{13}$ These comparisons with the U.S. data should be taken only as course checks on goodness of fit for at least two reasons. First, the returns may not be comparable, because we are generating returns on securities with payoffs denominated in terms of the consumption good. In contrast, the empirical estimates are typically computed from data generated by a monetary economy and then the payoffs are deflated. See Lucas (1984), for example, for a discussion of some theoretical differences between monetary and non-monetary asset pricing models. Second, we are comparing sample moments and the standard errors on the sample estimates from the data may be quite large.
} 
the incomes of the two types of agents are highly correlated, the correlations between consumptions and returns are comparable and, in fact, are somewhat higher for the type 2 agents. However, for the models with low income correlation, the correlation between the consumptions of the agents with the largest stock holdings with the equity return is larger. This is especially true in Model 6.

\section{Retrospective Remarks and Conclusions}

One objective of this paper was to show that it was computationally feasible to depart from the complete-markets/representative-agent setup in multiperiod models, and study interesting frictions in financial markets by simulation.

A second objective was to examine the implications of portfolio constraints for two empirical puzzles in the macroeconomics/finance literature: (i) the low correlations between individual consumptions and aggregate income, and (ii) the historically large equity premium (and low return on riskless assets), compared to what is implied by models with complete financial markets. In our benchmark economy, calibrated using observed incomes of workers in durable and non-durable sectors, we found that the equilibrium consumptions and asset returns moved in the right direction in terms of explaining the above observations, but the change with respect to the complete markets economy without constraints on asset holdings was not large: individual consumptions are highly correlated with aggregate income, the average riskless interest rate is too high, and the equity premium is too small.

Even though trading was constrained and markets incomplete, the features of constant relative risk averse preferences and the allowable trading in bonds provided agents with sufficient consumption smoothing opportunities for them to approximate the consumption plans that would be pursued with complete markets. Consumers sell (buy) some of their assets in bad (good) times. Therefore, under the benchmark case of Model 1, the 'precautionary motive' for holding assets discussed in Deaton (1989) is sufficient for equilibrium variables in the incomplete markets economy to behave similarly to the complete markets.

Also, since agents accumulate assets in such a way that borrowing constraints are rarely binding, the Euler equations are satisfied with equality for almost all periods. Therefore, despite the fact that the possibility of future binding constraints affects asset holdings in all periods, assets are almost always priced by the same equation as if constraints were not present. In the 
benchmark case, incomplete markets and borrowing constraints are incapable of explaining quantitatively the consumption-income correlation puzzle, and they only partially explain the equity premium puzzle.

Through experimentation with the individual income processes, we were able to identify some economic environments in which these puzzles could be resolved. Specifically, in Model 6, we assume a low correlation between the innovations to individual incomes (.17), comparably high volatilities of incomes, and a state-dependent borrowing constraint that precludes borrowing more than .33 of one's income. The high volatilities of incomes imply that the agents with relatively low income on average (type 2 agents) are frequently at or near their borrowing constraints. As such, trading in bonds is less effective as a means of smoothing consumption and type 2 agents must rely more heavily on equity trading to smooth consumption. Since type 2 agents find bonds relatively unattractive (compared to agents in economies with lower income variability), the mean of the riskless interest rate must be lower to induce agents to be comfortable with lower bond holdings. In this manner we are able to match the historically low average yield on riskless bonds, and to generate a sizable equity premium of approximately $4.6 \%$. This suggests that the two puzzles are intimately interconnected.

Furthermore, the environment with low income correlation and high income volatility gives individual consumptions that are relatively weakly correlated with aggregate income. However, these low correlations are achieved at the expense of relatively high individual consumption volatilities. Indeed, the standard deviations of individual consumptions and incomes are comparable for each type of agent and, in this sense, the binding borrowing constraints substantially limit agents' ability to smooth consumption. This highlights the extreme nature of this parameterization.

All of these conclusions must be considered tentative, pending further exploration of the qualitative properties of models with heterogeneous incomes and borrowing and short-sale constraints. Three qualifications in particular deserve mention. First, existence of an equilibrium for our economies with continuous state spaces and state-dependent constraints has not yet been established. Second, all of these calculations are for the case of firstorder approximations to the conditional expectations appearing in agents' Euler equations: expectations were assumed exponentials of affine functions of the state vector. We have experimented with exponential functions of second-order polynomials and found qualitatively similar results for most correlations.

However, our experience with second-order approximations leads to our third qualification. Namely, the values of certain simulated sample moments were quite sensitive to a number of small variations in the way the equilibrium 
was computed: after varying either the starting values for the iterations $\beta^{0}$, or the accuracy of the fixed point, or the number of observations $T$, or the degree of the polynomial, the algorithm iterated for a long time, making very small steps at a time, settling down finally at a new fixed point where some properties of the model changed. This was particularly true of the means of the equilibrium consumptions and asset holdings.

It is known that, if there exists a unique ergodic solution to the model, then $P E A$ will approximate this solution arbitrarily well with a high enough polynomial degree. ${ }^{14}$ Therefore, there are a number of reasons why we might be having difficulties in our solution. First of all, we have questions in our mind about whether the equilibrium consumptions and investments are ergodic. ${ }^{15}$ To our knowledge, this is a largely unexplored issue in the literature. While sufficient conditions on the equilibrium law of motion can be imposed to assure ergodicity, along the lines of the damping conditions in Duffie and Singleton (1993), we have not been successful at showing that these conditions are satisfied in theory by our model. In addition, it is doubtful that the equilibrium is unique ${ }^{16}$; again, this is a largely unexplored issue in the literature. This leads to our next largely unexplored issue: what is the 'correct' set of state variables for incomplete markets economies?. We, and other researchers, decided to use wealth as the only endogenous state variable but, presumably, other equilibria could be constructed including additional state variables that summarize past shocks in a different way.

If equilibria were non-unique, non-ergodic and several state vectors could be chosen, any algorithm would have difficulties, since it might wander around among the many valid solutions to the model. These problems would manifest themselves in the $P E A$ because the simulations generated in Step 2 of the algorithm would change slightly from one iteration to the next, which is exactly what happens in our calculations.

At this juncture, these observations remain concerns. We cannot say with confidence that any of them are or are not present. Accordingly, we view our findings as tentative, pending a more in-depth exploration of the properties of the equilibria of dynamic models with incomplete markets and state-dependent borrowing and short-sale constraints.

\footnotetext{
${ }^{14}$ See Marcet and Marshall (1994).

${ }^{15}$ After all the complete markets economy is non-ergodic in the following sense: under complete markets $c_{1 t} / c_{2 t}=\lambda$ for all $t$, and $\lambda$ is different for economies with different initial wealth. Therefore, a different initial individual wealth causes a permanent effect on the ratio of individual consumption, and the process $\left\{\left(c_{1 t}, c_{2 t}, y_{t}, w_{1 t}, w_{2 t}\right\}\right.$ is non-ergodic under complete markets. To the extent that the incomplete markets economy behaves much like the complete markets economy, we would expect it to be non-ergodic as well.

${ }^{16}$ In fact, some papers, including Duffie et al. (1994), construct incomplete market equilibria by comparing them to bubble equilibria, which are well known to be non-unique.
} 


\section{References}

[1] Breeden, D., 1989, "Optimal Dynamic Trading Strategies: A Simple Approach," manuscript, Duke University.

[2] Bossaerts, P and R. C. Green, 1989, "A General Equilibrium Model Of Changing Risk Premia: Theory And Tests" Review of Financial Studies 4 (2), pp. 467-494.

[3] Carroll, C. and L. Summers, 1991, "Consumption Growth Parrells Income Growth: Some New Evidence", in National Saving and Economic Performance, ed. by B. Bernheim and J. Shoven, Chicago University Press, Chicago.

[4] Cox, J. and C. Huang, 1987, "Optimal Consumption and Portfolio Policies when Asset Prices Follow a Diffusion Process," manuscript, MIT.

[5] Deaton, A., 1989, "Savings and Liquidity Constraints," NBER working paper No. 3196.

[6] den Haan and Marcet, 1990; "Solving a Simple Growth Model by Parameterizing Expectations" Journal of Business and Economic Statistics, January, 31-34.

[7] Duffie, D., J. Geanakoplos, A. Mas-Colell, and A. McClennan, 1994, "Stationary Markov Equilibria", Econometrica, vol. 62, pp. 745-782.

[8] Duffie, D. and K. J. Singleton, 1993, "Simulated Moments Estimation of Markov Models of Asset Prices", Econometrica, vol. 61, pp. 929-952.

[9] Flavin, M., 1981, "The Adjustment of Consumption to Changing Expectations about Future Income," Journal of Political Economy 89, 9741009.

[10] Hall, R.E. and F.S. Mishkin, 1982, "The Sensitivity of Consumption to Transitory Income: Estimates from Panel Data on Households," Econometrica 50, 461-81.

[11] He, H. and N. Pearson, 1989a, "Consumption and Portfolio Policies with Incomplete Markets and Short-sale Constraints: The Finite Dimensional Case," manuscript, UC-Berkeley.

[12] He, H. and N. Pearson, 1989b, "Consumption and Portfolio Policies with Incomplete Markets and Short-sale Constraints: the Infinite Dimensional Case," manuscript, UC-Berkeley.

[13] Ibbotson, R.G., 1989 "Stocks, Bonds, Bills and Inflation" Yearbook, Ibbotson Associates, Chicago. 
[14] Karatzas, Lehoczky, and Shreve, 1989, "Existence and Uniqueness of Multi- Agent Equilibrium in a Stochastic Dynamic Consumption/Investment Model," forthcoming, Math. Operations Research.

[15] Ketterer, J. and A. Marcet, 1990, "Introduction of Derivative Securities: A General Equilibrium Approach," manuscript, Carnegie Mellon University.

[16] Lucas, R. E. Jr., 1978, "Asset Prices in an Exchange Economy," Econometrica 26, 1429-1445.

[17] Lucas, R. E. Jr., 1984, "Money in a Theory of Finance," in K. Brunner and A. Meltzer, eds., Carnegie-Rochester Conference Series on Public Policy, North Holland: Amsterdam.

[18] MaCurdy, T.E., 1982, "The Use of Time Series Processes to Model the error Structure of Earnings in Longitudinal Data Analysis," Journal of Econometrics 18, 83-114.

[19] Mankiw, N. G., 1986, "The Equity Premium and the Concentration of Aggregate Shock," Journal of Financial Economics 17, 211-219.

[20] Mankiw, N. G. and S. Zeldes, 1990,"The Consumption of Stockholders and Non-stockholders," NBER working paper.

[21] Marcet, A., 1989, "Solution of Nonlinear Models by Parameterizing Expectations," manuscript, Carnegie Mellon University.

[22] Marcet, A. and D. Marshall, 1994, "Solving Nonlinear Rational Expectations Models by Parameterized Expectations: Convergence to Stationary Solutions", working paper, Universitat Pompeu Fabra and Federal Reserve Bank of Minneapolis.

[23] Marshall, D., 1990, "Solving Nonlinear Models by Parameterizing Expectations: Convergence Results," WP No. 73, Kellogg School, Northwestern University.

[24] Mehra, R. and E. Prescott, 1985, "The Equity Premium: A Puzzle," Journal of Monetary Economics 10, 335-359.

[25] Singleton, K.J., 1993, "Econometric Analysis of Representative Agent Intertemporal Asset Pricing Models", in Advances in Econometrics, Sixth World Congress, ed. C. A. Sims, Cambridge University Press.

[26] Tauchen, G., 1985, "Finite State Markov Chain Approximations to Univariate and Vector Autoregressions," Economic Letters 20, 177-181.

[27] Taylor, J. and H. Uhlig, 1990, "Solving Nonlinear Stochastic Growth Models: A Comparison of Alternative Solution Methods," Journal of Business and Economic Statistics 8, 1-50. 
[28] Telmer, C., 1990, "Asset Pricing Puzzles and Incomplete Markets," manuscript, Queen's University.

[29] Weil, P., 1989, "The Equity Premium Puzzle and the Riskfree Rate Puzzle," Journal of Monetary Economics 24, 401-421.

[30] Zeldes, S., 1989, "Consumption and Liquidity Constraints: An Empirical Investigation," Journal of Political Economy 97, 305-46. 


\section{APPENDIX 1}

Here we write the whole system (13) for our model. This is the system that delivers $\left\{z_{t}(\beta)\right\}$, although the dependence on $\beta$ is left implicit in the equations below. Notice that we introduce the Euler equation for the stock rewritten so as to insure invertibility, as discussed in section 3.2.

$$
\begin{aligned}
& \text { either } \\
& \mathrm{mu}_{i}\left(c_{i t}, \gamma_{i}\right) p_{t}^{b}=\delta_{i} P i_{t}^{b} \quad \text { and } b_{i t}>K_{i}^{b}\left(x_{t}\right) \\
& \text { or } \\
& \operatorname{mu}_{i}\left(c_{i t}, \gamma_{i}\right) p_{t}^{b} \geq \delta_{i} P i_{t}^{b} \quad \text { and } b_{i t}=K_{i}^{b}\left(x_{t}\right) ; \\
& p_{t}^{s} \mathcal{G}\left(a_{1 t}^{s}\right)=\delta P 1_{t}^{s} \quad \text { and } a_{1 t} \geq K_{1}^{s}\left(x_{t}\right) \\
& \text { or } \\
& p_{t}^{s} \mathcal{G}\left(a_{1 t}^{s}\right) \geq \delta P 1_{t}^{s} \quad \text { and } a_{1 t}=K_{1}^{s}\left(x_{t}\right) ;
\end{aligned}
$$

either

$$
\begin{aligned}
\mathrm{mu}_{2}\left(c_{2 t}, \gamma_{2}\right) p_{t}^{s} & =\delta_{i} P 2_{t}^{s} \quad \text { and } a_{2 t}>K_{2}^{s}\left(x_{t}\right) \\
\begin{array}{c}
\text { or } \\
\mathrm{mu}_{2}\left(c_{2 t}, \gamma_{2}\right) p_{t}^{s}
\end{array} & \geq \delta_{2} P 2_{t}^{s} \quad \text { and } a_{2 t}=K_{2}^{s}\left(x_{t}\right) ; \\
c_{1 t}+p_{t}^{s} a_{1 t}+p_{t}^{b} b_{1 t} & =a_{1 t-1}\left(p_{t}^{s}+d_{t}\right)+b_{1 t-1}+y_{1 t} \\
\pi c_{1 t}+(1-\pi) c_{2 t} & =y_{t}^{*} \\
\pi a_{1 t}+(1-\pi) a_{2 t} & =1 \\
\pi b_{1 t}+(1-\pi) b_{2 t} & =0
\end{aligned}
$$

Notice that these are eight equations (since (28) holds for $i=1,2$ ). 
Table 1: Description of Models

\begin{tabular}{cccccccc} 
Model & $A_{12}$ & $\rho_{y_{1} y_{2}}$ & $\sigma_{\epsilon_{1}}$ & $\sigma_{\epsilon_{2}}$ & $\rho_{\epsilon_{1} \epsilon_{2}}$ & $K_{i}^{b}$ & $K_{i}^{s}$ \\
\hline 1 & .15 & .78 & .05 & .03 & .5 & $-.33 y_{i}$ & .0 \\
2 & .15 & .78 & .05 & .03 & .5 & $-.5 y_{i}$ & .0 \\
3 & .15 & .78 & .05 & .03 & .5 & $-.5 y_{i}$ & .25 \\
4 & .0 & .13 & .05 & .03 & .2 & $-.33 y_{i}$ & .0 \\
5 & .0 & .13 & .05 & .03 & .2 & -.33 & .0 \\
6 & .0 & .17 & .05 & .05 & .2 & $-.33 y_{i}$ & .0
\end{tabular}

Table 2: Sample Moments of Consumptions and Incomes

\begin{tabular}{ccccccccc} 
Model & $\mu_{c_{1}}$ & $\sigma_{c_{1}}$ & $\mu_{c_{2}}$ & $\sigma_{c_{2}}$ & $\rho_{c_{1} y_{1}}$ & $\rho_{c_{1} y^{*}}$ & $\rho_{c_{2} y_{2}}$ & $\rho_{c_{2} y^{*}}$ \\
\hline 1 & 1.26 & .141 & .887 & .081 & .988 & .993 & .927 & .953 \\
2 & 1.28 & .142 & .854 & .079 & .987 & .994 & .917 & .955 \\
3 & 1.23 & .138 & .932 & .084 & .987 & .994 & .917 & .967 \\
4 & 1.24 & .086 & .902 & .078 & .957 & .930 & .855 & .793 \\
5 & 1.25 & .084 & .905 & .075 & .959 & .938 & .830 & .812 \\
6 & 1.20 & .088 & .975 & .130 & .947 & .843 & .939 & .834
\end{tabular}

Table 3: Sample Moments of Asset Returns

\begin{tabular}{cccccccc} 
Model & $\mu_{r s}$ & $\sigma_{r^{s}}$ & $\mu_{r^{b}}$ & $\sigma_{r^{s}}$ & $\mathrm{EP}=\mu_{r s}-\mu_{r^{b}}$ & $\rho_{c_{1} r^{s}}$ & $\rho_{c_{2} r^{s}}$ \\
\hline 1 & .051 & .062 & .020 & .020 & .031 & .157 & .175 \\
2 & .055 & .059 & .021 & .023 & .034 & .154 & .170 \\
3 & .040 & .065 & .026 & .017 & .027 & .163 & .175 \\
4 & .045 & .061 & .022 & .019 & .027 & .221 & .188 \\
5 & .050 & .061 & .021 & .019 & .029 & .221 & .193 \\
6 & .052 & .065 & .008 & .023 & .046 & .202 & .148
\end{tabular}

Notes:

$A_{12}$ is the $(1,2)$ element of the autoregressive matrix $A$.

Asset returns are defined as $r_{t}^{s} \equiv \frac{p_{t+1}^{s}+d_{t+1}}{p_{t}^{s}}$ and $r_{t}^{b} \equiv 1 / p_{t}^{b}$.

In all models, $\gamma=-2$. and $\delta=.99$.

$\sigma_{X}, \mu_{X}, \rho_{X, Y}$ represent, respectively, the standard deviation, mean, and correlation of random variables $X_{t}, Y_{t}$. 